\title{
Síndrome de Lennox-Gastaut: relato de caso
}

\author{
Lennox-Gastaut Syndrome: case report \\ Síndrome Lennox-Gastaut: reporte de un caso \\ Helinaldo Corrêa da CONCEIÇÃO ${ }^{1}$ \\ Alessandra Valle SALINO ${ }^{2}$ \\ Ana Karoline de Souza QUEIROZ ${ }^{3}$ \\ Eliane de Oliveira Aranha RIBEIRO ${ }^{4}$ \\ Keuly Sousa SOARES ${ }^{4}$ \\ Gimol Benchimol de Resende PRESTES ${ }^{5}$ \\ ${ }^{1}$ Acadêmico do Curso de Graduação em Odontologia, Escola Superior de Ciências da Saúde, \\ Universidade do Estado do Amazonas, UEA, 69065-001 Manaus-AM, Brasil \\ ${ }^{2}$ Doutoranda em Saúde Coletiva, Instituto de Medicina Social, \\ Universidade do Estado do Rio de Janeiro, IMS-UERJ, 20550-013 Rio de Janeiro - RJ, Brasil \\ Professora da Escola Superior de Ciências da Saúde, Universidade do Estado do Amazonas, UEA, 69065-001 Manaus-AM, Brasil \\ ${ }^{3}$ Cirurgiã-Dentista, Escola Superior de Ciências da Saúde, Universidade do Estado do Amazonas, UEA, 69065-001 Manaus-AM, Brasil \\ Pós Graduanda Lato Sensu em Odontologia para Pacientes com Necessidades Especiais, \\ Universidade do Estado do Amazonas, UEA, 69065-001 Manaus-AM, Brasil \\ ${ }^{4}$ Professora da Escola Superior de Ciências da Saúde, Universidade do Estado do Amazonas, UEA, 69065-001 Manaus-AM, Brasil \\ Especialista em Pacientes Portadores de Necessidades Especiais - Conselho Federal de Odontologia - CFO \\ ${ }^{5}$ Professora da Escola Superior de Ciências da Saúde, Universidade do Estado do Amazonas, UEA, 69065-001 Manaus-AM, Brasil \\ Doutora - Programa de Pós-Graduação em Odontologia, Área de Concentração em Odontopediatria, \\ Universidade Federal de Santa Catarina, UFSC 88040-900, Florianópolis-SC, Brasil
}

\begin{abstract}
Resumo
A síndrome de Lennox-Gastaut (SLG) é uma encefalopatia epilética severa da infância que corresponde a 5\% das epilepsias infantis, com início entre 1 e 8 anos de idade. Caracteriza-se por retardo mental progressivo, crises de múltiplos tipos. A etiopatogenia é obscura, sugerindo reação inespecífica de lesão cerebral e tratamento, geralmente ineficaz, com uso de valproato e benzodiazepínicos. O prognóstico é ruim, com pequeno número de pacientes com controle das crises. O objetivo do presente trabalho é apresentar as características presentes na SLG e a importância de tratamento individualizado para cada paciente, através do relato de caso clínico. Paciente IBC do gênero feminino, 33 anos, com diagnóstico de SLG logo ao nascimento, após a primeira crise convulsiva. Foi atendida na Clínica de Pacientes Especiais da Universidade do Estado do Amazonas (UEA) com queixa principal de cárie nos incisivos inferiores. Inicialmente a paciente apresentava trauma no incisivo central (11) durante crise epilética ocorrido na infância. Foi realizado exame clínico e o plano de tratamento restauração dos elementos 31 e 41, aplicação de flúor e orientação de higiene oral. O cirurgião-dentista deve estar preparado para o atendimento das pessoas com deficiência por meio da capacitação técnica e cultivo de valores humanos.
\end{abstract}

Descritores: Síndrome de Lennox Gastaut; Epilepsia; Etiologia; Prevalência; Encefalopatias.

\begin{abstract}
Lennox-Gastaut syndrome (SLG) is a severe childhood epilepsy encephalopathy that corresponds to 5\% of childhood epilepsies, beginning between 1 and 8 years of age. It is characterized by progressive mental retardation, crises of multiple types. The etiopathogenesis is obscure, suggesting nonspecific brain injury and generally ineffective treatment with valproate and benzodiazepines. The prognosis is poor, with a small number of patients with seizure control. The objective of the present study is to present the characteristics present in SLG and the importance of individualized treatment for each patient, through the clinical case report. Female patient IBC, 33 years old, diagnosed with of SGA at birth after the first seizure. She was attended at the Special Patients Clinic of the University of the State of Amazonas (UEA) with primary complaint of caries in the lower incisors. Initially, the patient presented trauma to the central incisor (11) during an epileptic crisis that occurred in childhood. The clinical examination and treatment plan of the restoration of elements 31 and 41, fluoride application and oral hygiene orientation were performed. The dentistry must be prepared for the care of people with disabilities through technical training and cultivation of human values.

Descriptors: Lennox Gastaut Syndrome; Epilepsy; Etiology; Prevalence; Brain Diseases.
\end{abstract}

\section{Resumen}

El síndrome de Lennox-Gastaut (LG) es una grave encefalopatía epileptiforme de la infancia que corresponde al 5\% de los desórdenes convulsivos, comenzando entre 1 y 8 años. Se caracteriza por retraso mental progresivo, varios tipos de convulsiones. La patogénesis es claro, lo que sugiere reacción no específica de la lesión cerebral y el tratamiento, por lo general ineficaces, con el uso de valproato y las benzodiacepinas. El pronóstico es malo, con un pequeño número de pacientes con control de las convulsiones. El objetivo de este trabajo es presentar las características presentes en la LG y la importancia de un tratamiento individualizado para cada paciente a través de la presentación de un caso. IBC paciente de sexo femenino, de 33 años de edad, con diagnóstico de LG al nacer, después del primer ataque. El paciente buscó la Clínica para Pacientes Especiales de la Universidad del Estado de Amazonas (UEA) quejándose de la caries en los incisivos inferiores. Inicialmente, el paciente tenía un traumatismo en el incisivo central (11) durante la crisis epiléptica ocurrido en la infancia. Se lleva a cabo plan de inspección y restauración de los elementos 31 y 41, la aplicación de flúor y la orientación para la higiene bucal. El dentista debe estar preparado para la atención de las personas con discapacidad a través de la capacitación técnica y el cultivo de los valores humanos

Descriptores: Síndrome de Lennox Gastaut; Epilepsia; Etiología; Prevalencia; Encefalopatías.

\section{INTRODUÇÃO}

Lennox e Davis foram os primeiros pesquisadores a estudarem a SLG 1950, e posteriormente foi estudada pelo francês Henri Gastaut e outros, em $1966^{1}$.
De maneira geral, essa síndrome, tem início na infância precoce surgindo entre o primeiro e o sexto ano de vida, porém alguns autores relatam até o sétimo e oitavo ano 
e suas anormalidades podem contribuir com disfunção progressiva. Assim sendo, essa é uma forma de epilepsia difícil de tratar, com crises diversificadas, acompanhadas por problemas comportamentais e retardo mental ${ }^{2}$.

Conforme estimativas, mais de um bilhão de pessoas vivem com algum tipo de deficiência, o que representa cerca de $15 \%$ da população mundial ${ }^{3}$. O Brasil possui aproximadamente $23,92 \%$ da sua população com algum tipo de deficiência, o que corresponde a aproximadamente 45,6 milhões de pessoas. Aproximadamente $18,8 \%$ deles são deficientes visuais; $7 \%$ com deficiência motora; $5,1 \%$ com deficiência auditiva, 1,4\% com deficiência mental ou intelectual. A maior parte desses indivíduos está na Região Nordeste $(26,3 \%)$. As menores incidências ocorreram nas regiões Sul e Centro Oeste, 22,5\% e 22,51\%, respectivamente, no estado do Amazonas 22,71\% da população tem algum tipo de deficiência ${ }^{4}$.

Dados da equipe da UEA comprovam que, em se tratando de diagnóstico das patologias de base, o maior percentual é de pacientes com distúrbios neurológicos (37,2\%), seguindo de distúrbios congênitos (16,6\%), múltiplas deficiências (12,6\%), distúrbios psicossociais $(12,1 \%)$, condições sistêmicas especiais $(11,3 \%)$, sem diagnóstico fechado $(4,2 \%)$, distúrbios sensoriais e áudiocomunicação $(3,2 \%)$ e deficiência física $(2,8 \%)^{5}$.

Os pacientes deficientes necessitam de um atendimento diferenciado por um determinado período ou por toda a sua vida. Por apresentarem certas limitações, muitos desses indivíduos podem não apresentar habilidade suficiente para realizarem higiene bucal de forma adequada e/ou eficiente ${ }^{6}$. Alguns pacientes apresentam problemas bucais relevantes e seus pais/responsáveis normalmente possuem dificuldades para encontrarem profissionais preparados para suas demandas. Dentre essas dificuldades destacam-se as barreiras arquitetônicas (são todas as limitações que as pessoas acometidas com alguma deficiência se deparam no seu dia a dia), as limitações financeiras, o medo e a ignorância/negligencia em relação à saúde ${ }^{7}$.

O tratamento odontológico em pacientes deficientes contribui para a melhoria da saúde geral e da qualidade de vida desses indivíduos, posto que a saúde bucal começa pela boca. Para tanto, a forma de abordar pacientes desse gênero é diferente de uma pessoa a qual não apresenta síndromes, pois o preparo dos profissionais envolvidos é um dos fatores determinantes para o sucesso do tratamento: o profissional deve cultivar valores humanos e estar apto a lidar com os pacientes e seus responsáveis ${ }^{8}$.

A prevenção, promoção da saúde bucal e o tratamento odontológico em pacientes especiais contribuem para a melhoria da saúde geral e da qualidade de vida desses indivíduos. A presença do biofilme bucal pode influenciar as terapêuticas médicas, devido aos fatores de virulência dos microrganismos que nela se encontram, os quais podem ser agravados pela presença de alterações bucais como a doença periodontal, cáries, necrose pulpar, lesões em mucosas, dentes fraturados ou infectados, traumas provocados por próteses fixas ou móveis, que podem repercutir na condição sistêmica do paciente ${ }^{9,10}$.

Segundo estudos, a SLG é uma síndrome rara, sua recorrência não possui uma relação direta com gênero ou classe social, porém não é detectado precocemente nas classes menos favorecidas, assim como o tratamento é menos acessível. A divulgação deste caso clínico é de grande importância no meio científico e social, servindo como base para direcionar o tratamento em situações semelhantes.

Com base no exposto o propósito deste trabalho é relatar um caso clínico odontológico de uma paciente com SLG, atendida na Disciplina de Clínica de Pacientes Especiais, do Curso de Odontologia, da Universidade do Estado do Amazonas evidenciando os aspectos clínicos gerais e bucais, com ênfase sobre o tratamento odontológico efetuado, dificuldades encontradas e aspectos familiares importantes.

\section{REVISÃO DA LITERATURA}

A síndrome de Lennox-Gastaut (SLG) é uma encefalopatia epilética severa da infância que corresponde a $5 \%$ das epilepsias infantis, descrita por Lennox e Davis em 1950, e posteriormente por Henri Gastaut e outros, em 1966. Foi definitivamente reconhecida durante o XIV Colóquio Europeu de Eletrencefalografia realizado em setembro de 1966 e, neste evento, por sugestão da filha de Lennox, o epônimo Lennox-Gastaut recebeu ampla aceitação. Henri Gastaut sugere que a prevalência da síndrome seja em torno de $6 \%$ de todas as epilepsias e cerca de $10 \%$ das epilepsias da infância ${ }^{1,2}$.

Trata-se de uma epilepsia grave de início infantil associada a uma deficiência intelectual e múltipla. Os pacientes apresentam atrofia cortical proeminente na região frontal mesial e polos temporais anteriores bilaterais, atrofia de substância branca generalizada e incluiu regiões pericentrais e pré-motoras. Atrofia proeminente na ponte, particularmente na região da formação reticular ${ }^{11}$.

A SLG é caracterizada por uma tríade de sintomas: retardo mental progressivo; múltiplas crises convulsivas de diversos tipos e de difícil controle; e eletroencefalograma (EEG) interictal constituído de complexos ponta e polipontaonda lenta (1 a $2 \mathrm{~Hz}$ ) difusos, com predomínio em regiões anteriores, atividades de base anormal e, em $70 \%$ dos casos, há "trens" de ponta rápida no sono não-REM. ${ }^{1,12}$. Acomete mais meninos do que meninas e corresponde a $4 \%$ dos pacientes com epilepsia da infância, com uma frequência de 0,26 a cada 1000 nascidos vivos nos Estados Unidos, a incidência anual na Europa é de 0,2-2,8 a cada 10000 nascimentos. A verdadeira prevalência nas diferentes populações não é conhecida, devido á disparidade entre os parâmetros utilizados para definir a doença, bem como a variabilidade dos fatores predisponentes entre diferentes populações $^{13 \text { e } 14}$.

Sabe-se que a doença apresenta seus primeiros sinais entre 1 e 8 anos de idade, tendo como pico as idades entre 1 e 3 anos. Em 30 a $50 \%$ dos casos, os pacientes mantêm as características clínicas da síndrome durante a idade adulta $^{15,16}$

As crises da SLG precisam de tratamento, mas muitas das vezes, essas crises, tornam-se refratárias, necessitando de múltiplas drogas em doses cada vez maiores, o que pode ser prejudicial, pois pode levar a um rebaixamento do nível de consciência e a um aumento paradoxal das crises. Assim sendo, as crises normalmente persistem por toda a vida do individuo adulto e tem um prognóstico ruim, apesar do tratamento farmacológico ser extensivo ${ }^{17}$.

Estudos epidemiológicos avaliando a prevalência da condição de cárie e doenças gengivais em pacientes com necessidades especiais demonstraram índices mais elevados em crianças com retardo mental, seguidos respectivamente de crianças com paralisia cerebral, cegueira, epilepsia, deficientes físicos, Síndrome de Down e surdos-mudos ${ }^{18}$. 
A falta de preparo dos cirurgiões-dentistas com relação à pacientes com deficiência ou retardo mental, conduzem a erros importantes de diagnóstico, levando os profissionais a assumir uma postura inadequada e um plano de tratamento errôneo ${ }^{19}$. O tratamento odontológico do paciente com necessidades especiais deve ser iniciado precocemente, assim que a sua condição sistêmica seja avaliada. Isto exige uma abordagem multidisciplinar, desafiando a formação técnica do cirurgião-dentista ${ }^{20}$.

O prognóstico da SLG é desfavorável e o controle das crises convulsivas ocorre apenas na minoria dos pacientes acometidos. A maioria evolui para crises parciais e encefalopatia epilética $^{1,21}$.

A importância do atendimento odontológico a pacientes especiais, enfatizando a adoção de medidas de promoção de saúde, assim como de atividades preventivas e curativas, sendo a interação dos pacientes com o profissional, a família e a sociedade importante para o sucesso do tratamento. Em sua pesquisa, o autor verificou que a negligência, no que diz respeito à saúde bucal destes pacientes, influencia o aumento das suas necessidades acumuladas. Portanto, torna-se fundamental conscientizar famílias, profissionais e os órgãos governamentais ${ }^{22}$.

A importância de assistência odontológica para pacientes com necessidades especiais, na qual seja incluído um programa de escovação supervisionada e educação para a saúde, voltado aos pais, cuidadores, e também aos alunos do curso de graduação em odontologia, professores e equipe de atenção multidisciplinar. No caso de pacientes que necessitam de atendimento ambulatorial, a orientação dietética, escovação supervisionada e aplicação tópica de flúor, devem ser medidas complementares adotadas. De acordo com os dados levantados pelos autores, observa-se que a maioria dos procedimentos executados durante o tratamento odontológico desses pacientes, está relacionada com a promoção da saúde mais do que procedimentos restauradores, indicando a viabilidade dessa abordagem em pacientes com necessidades especiais ${ }^{23}$

Um programa de controle mecânico e mecânico/químico de biofilme calcificado voltado aos pais são capazes de melhorar a saúde bucal de seus filhos. A maioria dos cuidadores relataram que com a orientação recebida, conseguiram superar as dificuldades para realizar ou auxiliar a escovação ${ }^{24,25}$.

A dieta cetogênica é um tratamento baseado em evidências para a epilepsia de difícil controle incluindo síndrome de Lennox-Gastaut. Esta dieta é baseada na ingestão de gordura de baixo carboidrato de alta proteína, ou seja, rica em lipídeos, moderada em proteínas e pobre em carboidratos $^{26}$.

Por outro lado sabe-se que, os traumatismos dentários são um fenômeno global, com frequência alta, chegando a 50 a $60 \%$, incluindo ambas as dentições. Embora as injurias traumáticas ocorram em qualquer idade, a idade mais comum em que afetam os dentes permanentes varia ativamente de 8 a $12 \operatorname{anos}^{27}$.

Estudos que descrevem a prevalência desses traumatismos em crianças com necessidades especiais demonstram que a porcentagem é de 18 a $28 \%{ }^{28,29}$. Dentre os pacientes especiais, os epiléticos estão mais susceptíveis aos traumatismos dentários. Em 1978 Bessemann demonstrou que $52 \%$ dos pacientes epiléticos apresentam lesões dentárias traumáticas, muitas vezes repetitivas. De maneira geral, esses traumatismos ocorrem principalmente devido a quedas ${ }^{30,31}$.
Tendo em vista que a síndrome de Lennox-Gastaut apresenta como característica básica a ocorrência de múltiplas crises convulsivas, a possibilidade de ocorrência de traumatismos dentários é elevada. No entanto, até o momento não há trabalhos publicados na literatura específica, relatando a ocorrência desse tipo de traumatismo em pacientes com síndrome de Lennox-Gastaut ${ }^{32}$.

Além disso, é importante que o cirurgião-dentista esteja presente na equipe de saúde que assiste pacientes com doenças raras, como a síndrome de Lennox-Gastaut, a fim de avaliá-los clínica e radiograficamente, estabelecendo e descrevendo os achados bucais pertinentes, que podem contribuir com o diagnóstico futuro de pacientes com a mesma síndrome. E, ainda, favorecendo o planejamento, o tratamento e o prognóstico do caso, do ponto de vista odontológico $^{32}$.

Trabalhos sugerem que $30 \%$ dos casos a SLG é de etiologia desconhecida. Em $60 \%$ dos casos, foram observados problemas neurológicos perinatais e pós-natais, incluindo a síndrome de West ${ }^{153}$. A prevalência é de 2 em 10.000 mil pessoas na Europa e existe predileção maior pelo gênero masculino ${ }^{34}$.

\section{CASO CLÍNICO}

A paciente IBC com síndrome de Lennox-Gastaut, 33 anos, gênero feminino, leucoderma, compareceu em 15 de março de 2016, acompanhada dos responsáveis à disciplina de Clínica de Pacientes Especiais da Universidade do Estado do Amazonas (UEA) com queixa principal de cárie nos incisivos inferiores.

Durante a anamnese foi perguntado aos pais sobre a história médica da paciente e mencionaram que a Síndrome foi diagnosticada por um médico neurologista, logo ao nascimento, após a primeira crise convulsiva. Além das crises, a paciente apresenta atraso no desenvolvimento intelectual e letargia, tanto no raciocínio quanto nos movimentos. A família teve um impacto com o diagnóstico, pois não era esperado ter uma filha com deficiência, em consequência disso, os pais apresentaram grande abalo emocional. Relataram que a paciente aos 10 anos teve uma crise epiléptica, em que caiu e fraturou o incisivo central superior esquerdo, por isso, o mesmo é escurecido. Os pais da paciente procuraram pela primeira vez tratamento odontológico para a filha em 1993, o local desse primeiro atendimento foi à clínica de odontologia da UFAM.

Caxumba foi à doença da infância, e além disso, aos 31 anos de idade, IBC passou por uma operação para a retirada da vesícula biliar, e desde então, os pais relataram que a filha apresenta problemas hepáticos como Gama-GT alterados, além de apresentar problemas de reações alérgicas à detergentes. Também foi relatado que a paciente apresenta problemas na coordenação motora, fala e locomoção o que a faz utilizar uma cadeira de rodas (Figura 1).

Segundo a mãe da paciente a alimentação da IBC é controlada, para tanto, carnes e frutas devem ser cortados em pequenos pedaços. Assim, mesmo tendo a dentição completa e os dentes em posições razoáveis, a paciente não consegue fazer a mastigação dos alimentos.

Para o controle das convulsões apresentadas pela paciente é feito o uso dos seguintes fármacos: Gardenal 100 mg, Tegretol $400 \mathrm{mg}$, Sabril $500 \mathrm{mg}$, Rivotril 0,5 mg e Topiramato $50 \mathrm{mg}$.

No exame clínico foi observado trauma nos dentes, principalmente nas incisais dos incisivos centrais superiores (11-21). Para o elemento 11 foi relatado trauma quando a 
paciente tinha 10 anos. No entanto, não foi possível fazer a tomada radiográfica para verificar se os dentes apresentavam reabsorção interna e também para fazer possíveis acompanhamentos, pois a paciente não colaborava.

Em razão da paciente não ser colaboradora foi necessário fazer contenção física/restrição física para execução dos procedimentos restauradores (Figura 2).

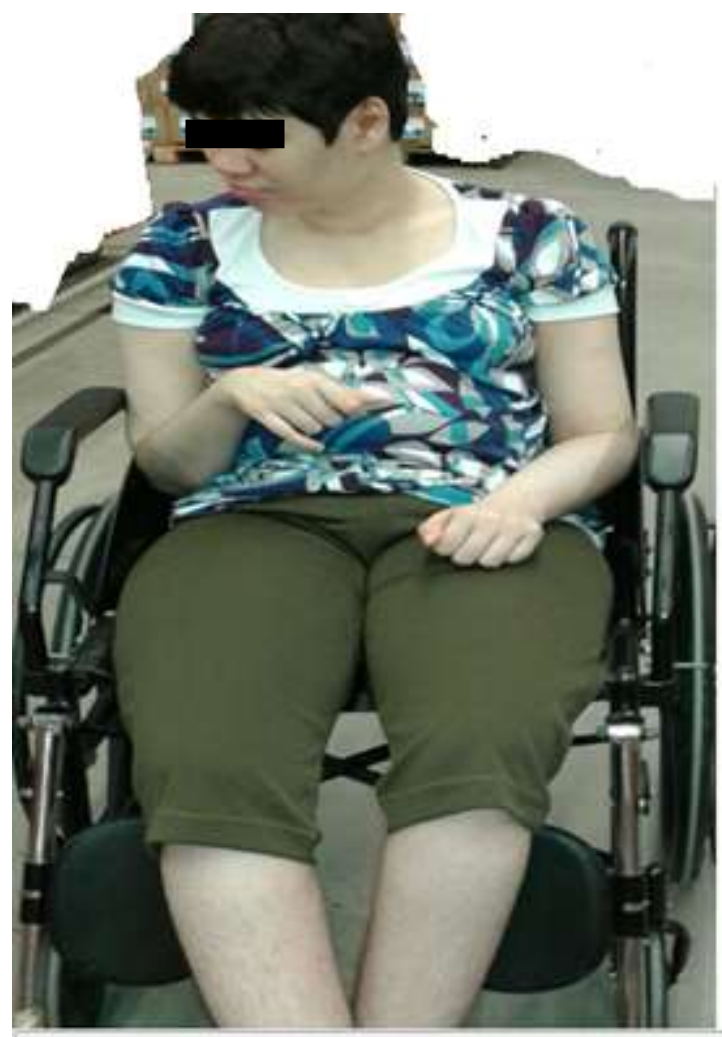

Figura 1: Paciente IBC mostrando sua condição especial

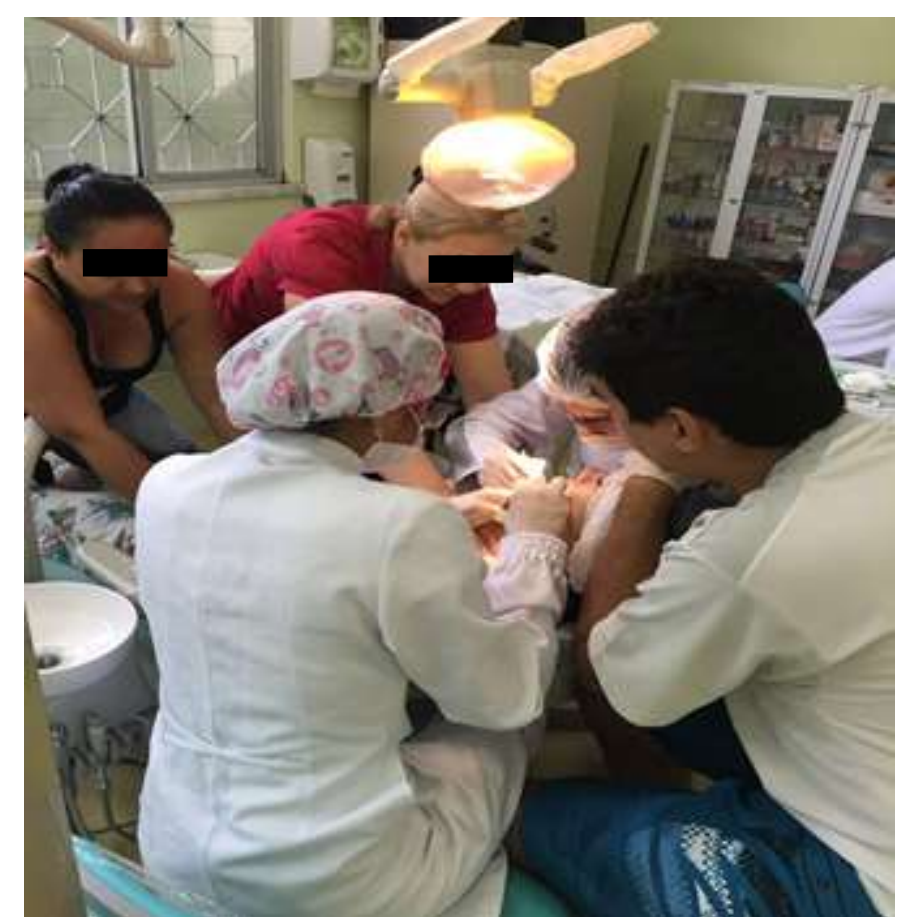

Figura 2: Restrição física realizada com ajuda dos cuidadores.

Verificou-se que o incisivo lateral superior (12) apresentava a face incisal traumatizada, bem como lesão de cárie nos incisivos centrais inferiores faces mesiais 31 e 41 classe IV de Black (Figuras 3, 4 e 5).

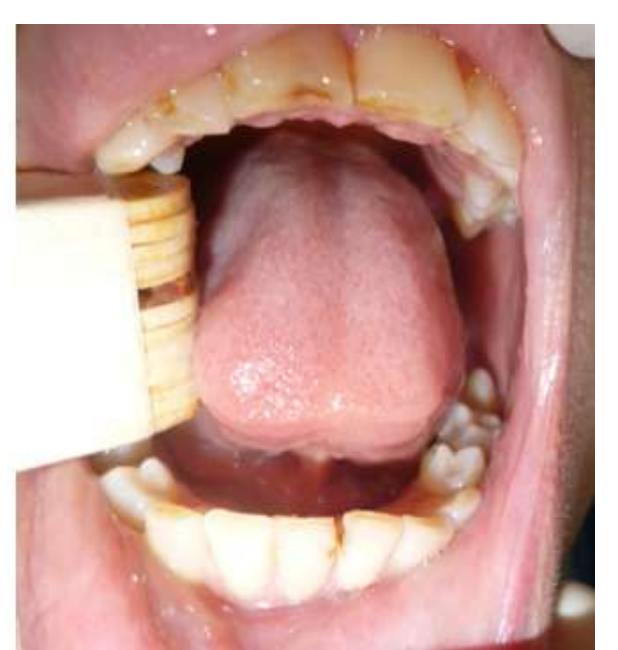

Figura 3: Desgaste na face incisal dos elementos dentários 11 e 21.

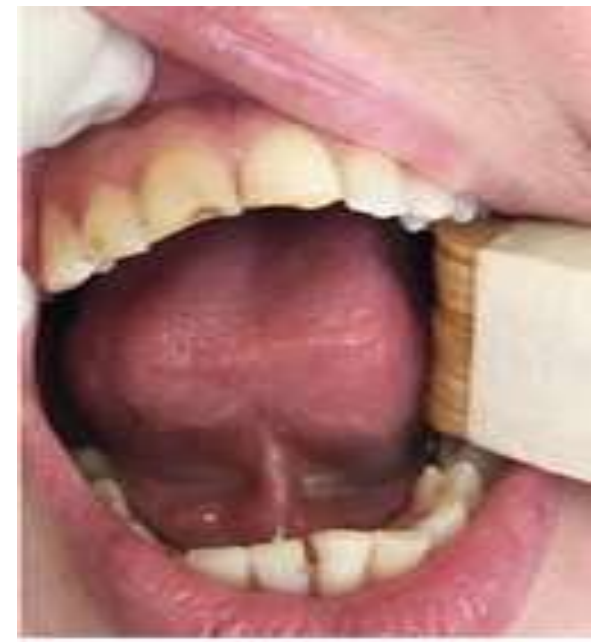

Figura 4: Desgaste na face incisal dos elementos dentários 11 e 21 .

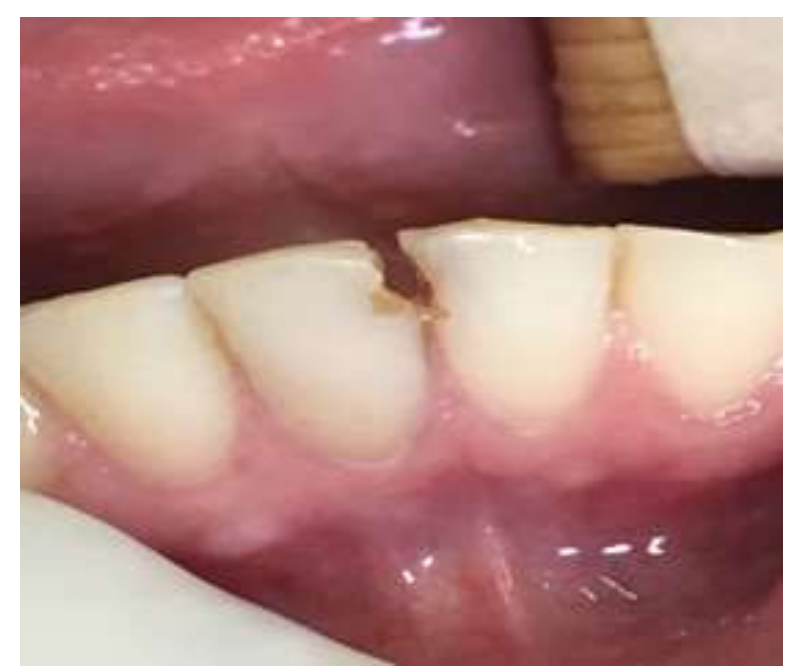

Figura 5: Lesão cariosa nos elementos dentários 31 e 41

Os elementos 31 e 41 foram restaurados com resina composta cor A3E (3M ESPE) e isolamento relativo com roletes de algodão, já que as dificuldades em pacientes especiais são muitas para realização do isolamento absoluto.

A profilaxia foi feita com pedra pomes e escova de Robson. Foi necessário usar um abridor de boca feito com palitos de madeira (Figura 6).

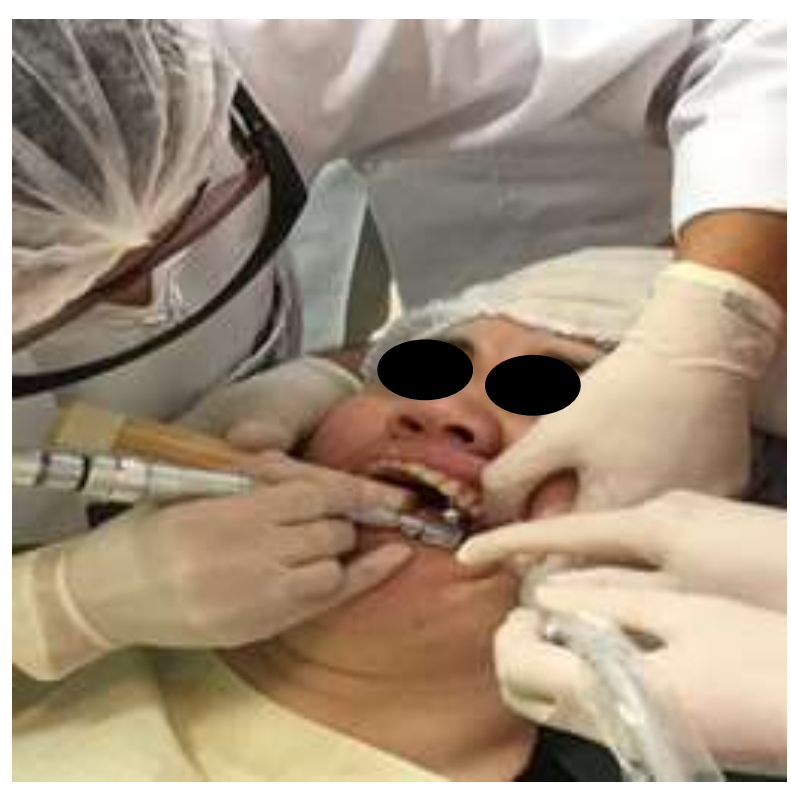

Figura 6: Profilaxia

Posteriormente a profilaxia foi iniciada a remoção da cárie dos elementos dentários (Figuras 7 e 8). As brocas usadas foram do tipo carbide multilaminadas em baixa rotação, além das colheres de dentina, de acordo com o tamanho da cavidade.

Em seguida, realizou-se o acabamento e o polimento das restaurações dos elementos 31 e 41, no acabamento inicial das restaurações por vestibular foram usadas brocas com pontas diamantadas de granulação fina, porém no polimento do terço vestíbulo-proximal foram usadas pontas abrasivas discoides (Sistema Enhance, Dentsply - Brasil) (Figuras 9 e 10). 


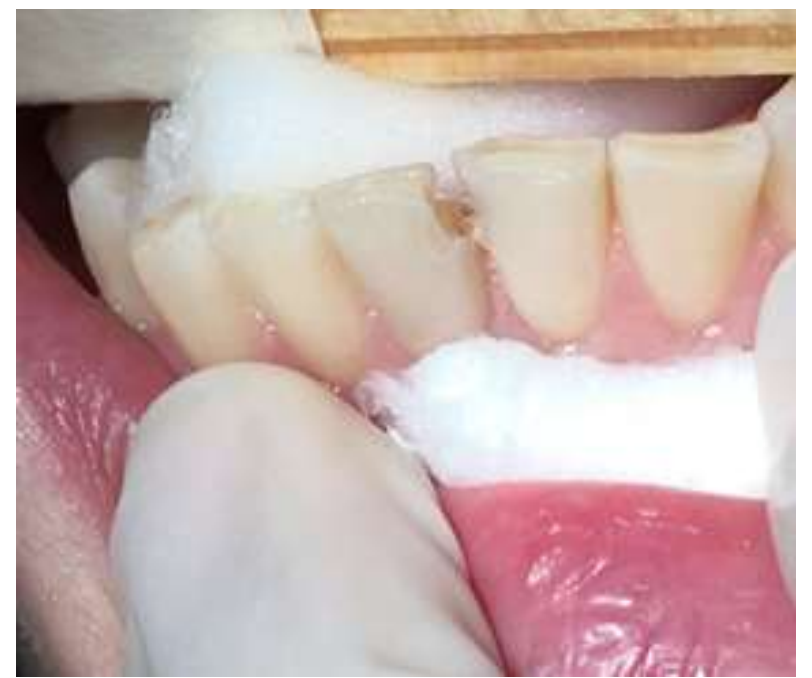

Figura 7: Preparo Cavitário.

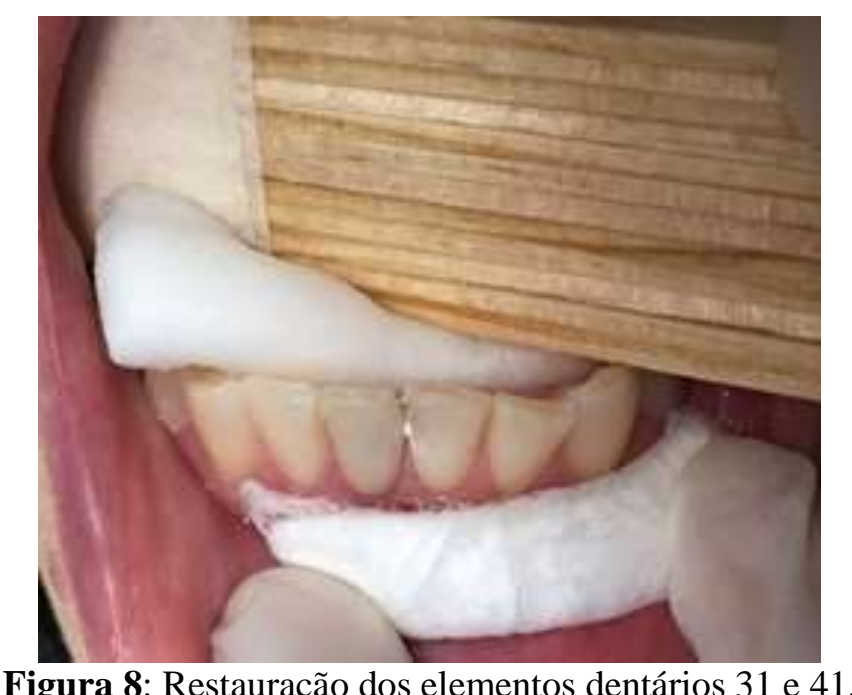

Figura 8: Restauração dos elementos dentários 31 e 41

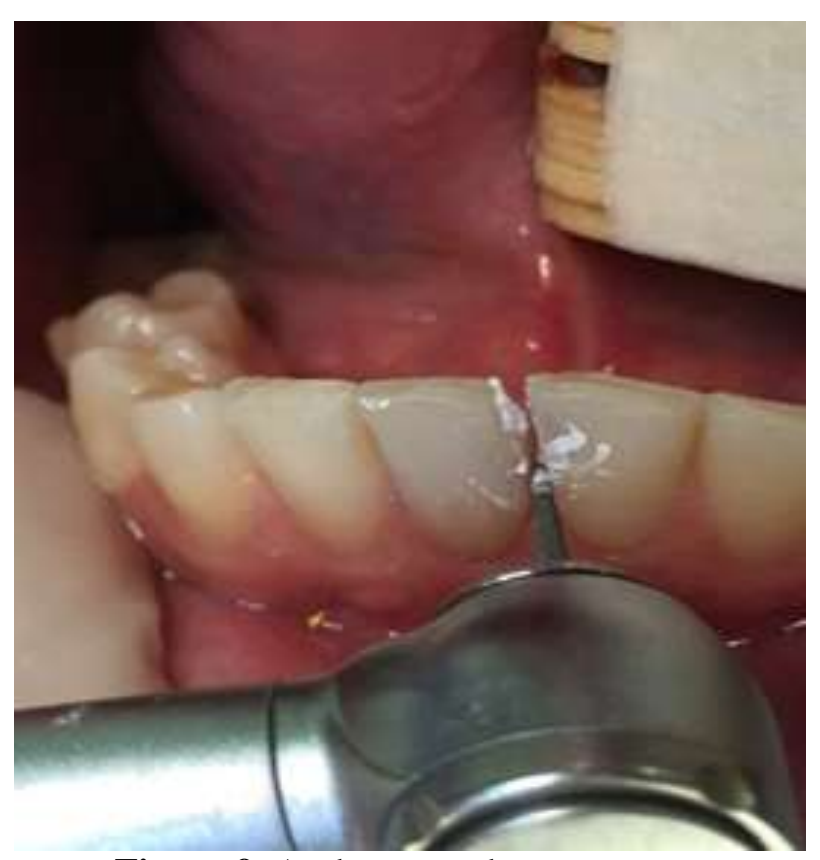

Figura 9: Acabamento da restauração.

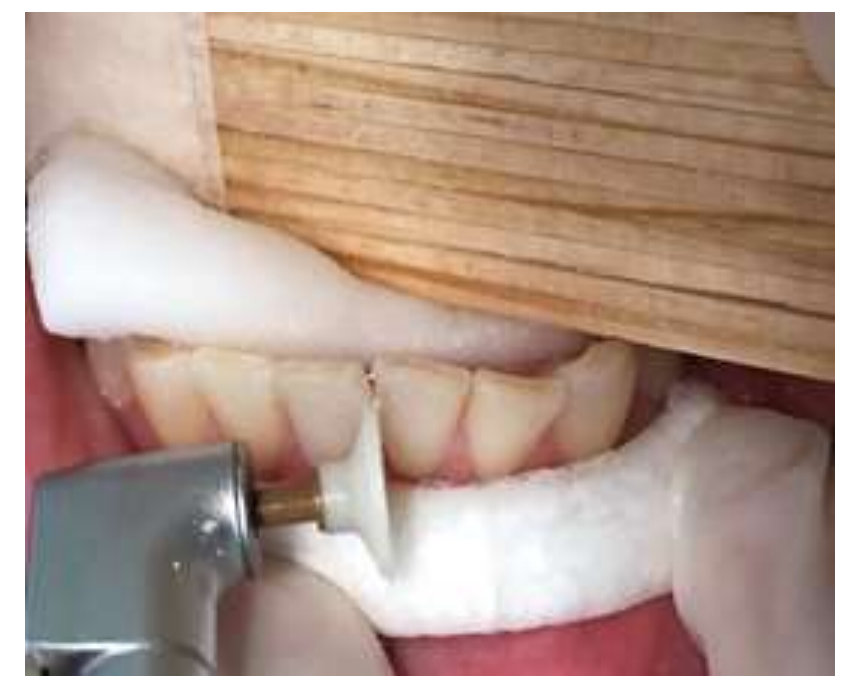

Figura 10: Polimento da restauração.

Para finalizar o procedimento odontológico, foi realizada aplicação de flúor, utilizou-se rolete de algodão (Figura 11).

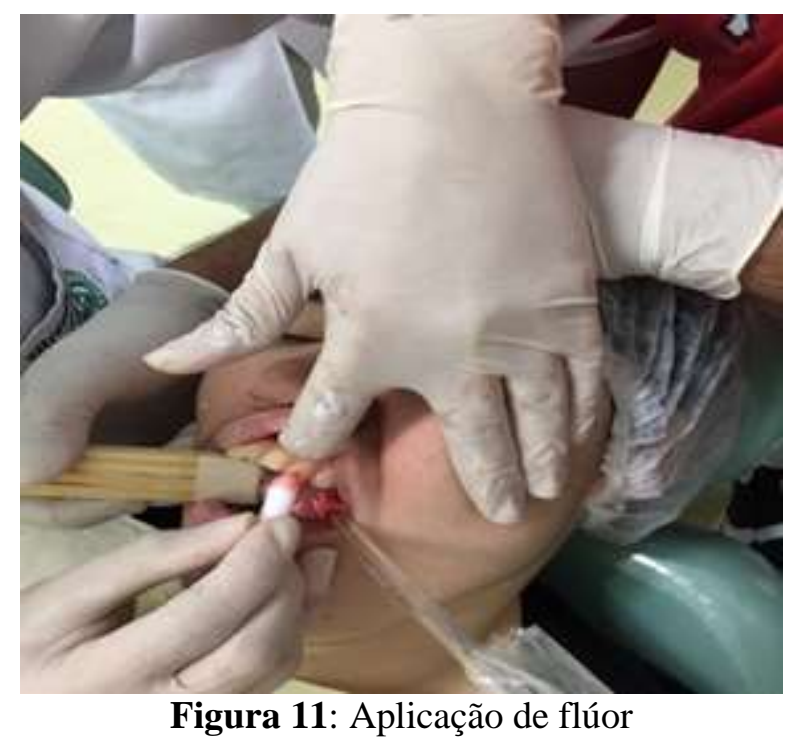

\section{DISCUSSÃO}

Os primeiros trabalhos a discutir achados bucodentais em paciente com síndrome de Lannox-Gastaut foram de Saleh e Stephen ${ }^{35}$. Nestes trabalhos encontraram: mordida aberta anterior, higiene precária com muito acúmulo de biofilme dental, lesões de cárie, doença periodontal com perda de altura de osso alveolar e gengivite generalizada ${ }^{35}$. Dentre esses achados bucodentais, os únicos que coincidem com os encontrados no presente caso são as lesões de cárie. No entanto, este é o primeiro caso onde se observou traumatismo dentário no incisivo lateral superior esquerdo elemento (11), porém compatível em pacientes que apresentam quadro convulsivo. Além disso, a paciente teve histórico de fratura de esmalte no incisivo lateral superior direito achado bucodental anteriormente descrito. As fraturas radiculares em dentes permanentes são relativamente raras, afetando cerca de 0,5 a $7 \%$ dos dentes traumatizados ${ }^{36-40}$.

Embora o incisivo central superior direito tenha tido alteração de cor, optamos por não realizar o tratamento endodôntico, pois para se justificar sua realização são necessários 2 ou mais sinais e sintomas clínicos e/ou radiográficos. No presente caso, o dente apresentava apenas um sinal, a alteração de cor. Por outro lado, a paciente não colaborava para a tomada radiográfica, assim sendo, essa é uma das principais dificuldades para a realização de tratamento endodôntico.

Um relato de caso clínico realizado na Bolívia encontrou na cavidade oral de um paciente masculino de 7 anos e 11 meses de idade: hipoplasia do esmalte, apinhamento dentário, presença de múltiplas cáries dentais e língua fissurada. Esse paciente recebeu tratamento integrado, a anestesia utilizada foi a geral $^{2}$.

No caso clínico descrito no presente trabalho, a paciente também apresentava hipoplasia do esmalte, apinhamento dentário e cáries em alguns dentes, porém não foi necessária anestesia geral, já que o procedimento era de baixa complexidade. No entanto, foi necessário lançar mão da contenção física/restrição física, realizada com ajuda dos responsáveis, para estabilizar a paciente na cadeira odontológica.

A falta de cuidados profiláticos e terapêuticos desencadeia graves problemas bucais em qualquer pessoa, seja ela normal ou deficiente. $\mathrm{O}$ organismo do paciente especial é comovido pelo desequilíbrio metabólico geral, onde o descuido gera lesões no organismo sendo a boca a cavidade onde se refletem as consequências deste descaso. Por isso, foi apresentada aos responsáveis da paciente orientação na técnica de escovação e também os tipos de escovas que deveriam ser usadas. Na mesma oportunidade, a mãe foi instruída quanto ao uso do fio dental para a paciente 
já que a paciente possui habilidade motora comprometida. Também recebeu orientação quanto a manter o acompanhamento odontológico para evitar possíveis consequências maiores.

$\mathrm{O}$ uso de drogas anticonvulsivantes causa maior propensão ao desenvolvimento de problemas periodontais ${ }^{41}$. Nossos achados bucais não são concordantes com essa afirmativa, uma vez que a paciente não apresentou gengivite generalizada. Pacientes especiais tendem a apresentar higiene bucal precária devido as suas limitações cognitivas e de coordenação motora. Por isso, Khatri ressalta a necessidade da conscientização dos pais e/ou responsáveis por esses pacientes da importância da realização da higiene bucal adequada, da realização de uma dieta não cariogênica, do uso consciente do fluoreto e do acompanhamento regular de um profissional ${ }^{42}$. Há a necessidade do controle do estresse e da ansiedade de pacientes que sofrem crises convulsivas.

\section{CONCLUSÃO}

O cirurgião-dentista deve estar preparado para o atendimento de pacientes com necessidades especiais por meio da capacitação técnica e cultivo de valores humanos, minimizando os diversos problemas que esses pacientes já estão condicionados a enfrentar devido ao seu estado de deficiência.

Em pacientes que sofrem de síndromes epiléticas, como a SLG, a probabilidade de ocorrência de quedas é elevada. É importante que o cirurgião-dentista esteja ciente desse risco aumentado.

Apesar de a paciente ser sindrômica os procedimentos realizados foram simples, pois a paciente faz acompanhamento odontológico periodicamente mantendo a saúde bucal.

\section{REFERÊNCIAS}

1. Wheless JW, Constantinou JE. Lennox-Gastaut syndrome. Pediatr Neurol, 1997; 17(3): 203-11.

2. Arce CC. Síndrome Lennox-Gastaut. Manifestaciones odontológicas. Rev. Soc Bol Ped, 2014; 53 (1): 21-3.

3. World Health Organization. Relatório mundial sobre a deficiência. The Word Bank; traduções Lexicus Serviços Linguísticos. São Paulo: SEDPcD,2012: 334p.

4. Instituto Brasileiro de Geografia e Estatística. Censo Demográfico 2010. Características gerais da população, religião e pessoas com deficiência. Rio de Janeiro: IBGE, 2010: 215.

5. Assis C. Dentista para lá de especiais. Rev Bras Odonto. 2014; 71(1): 58-61.

6. Resende VLS. Atendimento odontológico a pacientes com necessidades especiais. Anais do $8^{\circ}$ Encontro de Extensão da UFMG; 2005 Oct 03-04, Belo Horizonte, Brasil.

7. Jung LS. Serviços odontológicos oferecidos às crianças com necessidades especiais[ trabalho de conclusão do curso]. Porto Alegre: Universidade Federal do Rio Grande do Sul; 2011.

8. Moretto JM, Aguiar SMHCA, Alves Rezende MCR. Reflexões sobre a importância da assistencia odontológica preventiva e do adequado treinamento dos Cirurgiões-Dentistas para o atendimento de pessoas com deficiência. Arch Health Invest. 2014; 3(3): 58-64

9. Spalding M, Siqueira JTT. Avaliação de uma estratégia terapêutica em processos infecciosos bucodentais. RGO. 1999; 47(1): 110-4.
10. Pannuti CM, Lotufo RFM, Cai S, Saraiva MC, Freitas NM, Falsi D. Effect of a $0.5 \%$ chlorhexidine gel on dental plaque superinfecting microorganisms in mentally handicapped patients. Pesqui Odontol Bras 2003; 17(3): 228-33

11. Newham BJ, Curwood EK, Jackson GD, Archer JS. Pontine and cerebral atrophy in Lennox-Gastaut syndrome. Epilepsy Res. 2015; 120:98-103.

12. Bourgeois BFD, Douglass LM, Sankar R. LennoxGastaut Syndrome: A consensus approach to differential diagnosis. Epilepsia. 2014; 55(Suppl 4):4-9.

13. Gastaut H, Tassinari CA, Roger J, Soulayrol R, Saint Jean M, Regis H,et al. Epileptic encephalopathy in children with slow diffuse spike-wares (or petit mal variant) or Lennox syndrome. Recenti Prog Med. 1968; 45(2):117-46.

14. Heiskala H. Community-based study of Lennox-Gastaut syndrome. Epilepsia. 1997; 38(5):526-31.

15. Dulac O, N'Guyen T. The Lennox-Gastaut Syndrome. Epilepsia. 1993; 34(Suppl 7): S7-17.

16. Markand ON. Lennox-Gastaut syndrome (childhood epileptic encephalophaty). J Clin Neurophysiol. 2003; 20(6): 426-41.

17. Lancman G, Virk M, Shao H, Mazumdar M, Greenfield JP, Weinstein S, et al. Vagus nerve stimulation vs. corpus callosotomy in treatment of Lennox-Gastaut syndrome: a meta-analysis. Seizure. 2013;22(1): 3-8.

18. Gupta DP, Chowdhury R, Sarkar S. Prevalence of dental caries in handicapped childrens of Calcutta. J Indian Soc Pedod Prev Dent. 1993; 11(1): 23-7.

19. Oliveira ALBM, Giro EMA. Importância da abordagem precoce no tratamento odontológico de pacientes com necessidadades especiais. Odonto. $2011 ; 19(38)$ : 45-51.

20. Cançado Figueiredo M, Carvalho e Silva SR, Preto Guimarães F, Araújo VP. Perfil de pacientes com necesidades especiales. Bol Asoc Argent Odontol Niños. 2003; 32(1):8-11.

21. David P, Garcia V, Meneses S. Síndrome de LennoxGastaut, uma revisão atualizada. Revista Chilena de Epilepsia. 2014; 14(3):42-5.

22. Novaes MSP. Atenção odontológica integral a deficientes auditivos: uma proposta [tese]. São Paulo: Faculdade de Odontologia de da USP; 1997.

23. Abreu MHNG, Castilho LS, Resende VL. Assistência odontológica a indivíduos portadores de deficiências: o caso da Associação Mineira de Reabilitação e Escola Estadual João Moreira Salles. Arq Odontol. 2001;37(2): 153-61.

24. Abreu MHNG, Paixão HH, Resende VLS. Controle de placa bacteriana em portadores de deficiências físicas: avaliação de pais e responsáveis. Arq Odontol 1999; 35(1/2): 27-37.

25. Tomita NE, Fagote BF. Programa educativo em saúde bucal para pacientes especiais. Odontol Sociedade 1999, 1(1/2): 45-50.

26. De Lucia S, Pichard S, Ilea A et al. An unfortunate challenge: Ketogenic diet for the treatment of LennoxGastaut syndrome in tyrosinemia type 1. Eur J Paediatr Neurol. 2016; 20(4):674-7.

27. Glendor U, Marcenes W, AndreasenJ. Classification, epidemiology and etiology. In: JO, A.;FM, A., et al (Ed.). Textbook and color atlas of traumatic injuries to the teeth. 4 th. Oxford: Blackwell; 2007.

28. Nunn JH, Murray JJ. The dental health of handicapped children in Newcastle and Northumberland. Br Dent $\mathrm{J}$, 1987; 162(1): 9-14. 
29. Ohito FA, Opinya GN, Wang'ombe J. Traumatic dental injuries in normal and handicapped children in Nairobi, Kenya. East Afr Med. 1992; 69(12): 680-2.

30. Andreasen JO. Etiology and pathogenesis of traumatic dental injuries. A clinical study of 1,298 cases. Scand J Dent Res. 1970; 78(4):329-42.

31. Bessermann K. Frequency of maxilla-facial injuries in a hospital population of patients with epilepsy. Bull Nord Soc Dent Handicap. 1978, 5(1):12-26.

32. Pucinelli CM. Síndrome de Lennox Gastaut: Aspectos bucodentais relevantes para a Odontologia [trabalho de conclusão do curso]. Ribeirão Preto:FUNORP; 2015.

33. Ohtahara S. Lennox-Gastaut Syndrome. Considerations in its concept and categorization. J Psychiatry Neurol. 1988; 42(3):535-42.

34. Heiskala H. Community-based study of Lennox-Gastaut syndrome. Epilepsia. 1997;38(5):526-31.

35. Saleh AT, Stephen L. Case report Lennox Gastaut Syndrome, review of literature and a case report. Head \& Face Medicine 2008; 4(9): 1-7

36. Andreasen JO. Etiology and pathogenesis of traumatic dental injuries. A clinical study of 1,298 cases. Scand J Dent Res.1970; 78(4): 329-42.

37. Birch R, Rock WP. The incidence of complications following root fracture in permanent anterior teeth. $\mathrm{Br}$ Dent J. 1986;160(4):119-22.

38. Gelbier S. Injured anterior teeth in children. A preliminary discussion. Br Dent J. 1967;123(7):331-5.

39. Magnusson B, Holm AK. Traumatised permanent teeth in children-a follow-up. I. Pulpal complications and root resorption. Sven Tandlak Tidskr. 1969; 62(2): 6170.

40. Ravn JJ. Dental injuries in Copenhagen schoolchildren, school years 1967-1972. Community Dent Oral Epidemiol. 1974; 2(5):231-45.

41. Angelopoulos AP. A clinicopathological review. Diphenylhydantoin gingival hyperplasia: 2. A etiology, pathogenesis, differential diagnosis and treatment. Dent J. 1975; 41(5):275-83.

42. Khatri A. Dental findings in patients with West syndrome: a report of two cases. J Indian Soc Pedod Prev Dent. 2014; 32(2):168-71.

\section{CONFLITO DE INTERESSES}

Os autores declaram não haver conflitos de interesse.

\section{AUTOR PARA CORRESPONDÊNCIA}

Helinaldo Correa Conceição

helinaldouea@gmail.com

Submetido em 01/12/2016 Aceito em 01/01/2017 